\title{
Changes in levels of antioxidant markers and status of some enzyme activities among Falciparum Malaria Patients in Yemen
}

\begin{abstract}
Clinical data indicate that oxidative stress and activities may play important roles in Plasmodium falciparum. The aim of this study was to assess the erythrocyte antioxidants and evaluate enzyme activities as marker for Plasmodium falciparum malaria patients. The diagnosis of malaria was confirmed by thick and thin film with Giemsa staining of malaria parasite. Ninety consenting individuals, sixty infected patients and thirty uninfected subjects comprising both sexes were randomly selected and age range between 18 to 48 years. The levels of Erythrocytic superoxide dismutase eSOD, serum ceruloplasmin (CP), vitamin C, alkaline phosphatase (ALP) and lactate dehydrogenase (LDH) were determined. Low levels of essential antioxidants such as eSOD and vitamin $\mathrm{C}$ in the patients have been found to be associated with an increased risk of Plasmodium falciparum malaria. Low levels of antioxidants observed in the falciparum malaria patients may be due to their increased utilization to free radical scavenger. High level of CP observed in the Plasmodium falciparum malaria patients may be of powerful potency against oxidative stress. Furthermore, lowered levels of antioxidants especially of vitamin $\mathrm{C}$ in malaria infection suggest that antioxidant may play a major role in the treatment of malaria infection. It was detected that serum levels of ALP and LDH were significantly higher in Plasmodium falciparum malaria patients group, compared with the healthy group. Higher levels of LDH and ALP in the Plasmodium falciparum malaria patients may be used in the diagnosis and treatment and monitoring of patients with malaria. Results also suggest that the rise in LDH activity may be as a result of the cellular anoxia of liver rather than other tissues in malaria patients.
\end{abstract}

Keywords: Plasmodium falciparum, antioxidants, ceruloplasmin, superoxide dismutase, enzymeactivities
Volume 4 Issue 6 - 2017

\author{
Ali A Al Ezzi,' Mohamed B Al Salahy, ${ }^{2}$ Bushra \\ H Shnawa, ${ }^{3}$ Gamal H Abed, ${ }^{2}$ Ahmad M \\ Mandour $^{4}$ \\ 'Faculty of Science and Education, Aden University, Yemen \\ ${ }^{2}$ Faculty of Science, Assiut University, Egypt \\ ${ }^{3}$ Faculty of Science, Soran University, Iraq \\ ${ }^{4}$ Faculty of Medicine, Assiut University, Egypt
}

Correspondence: Ali A Al Ezzi, Department of Biology, Faculty of Science and Education, University of Aden, Yemen, Email dr.aliizy@gmail.com

Received: February 17, 2017| Published: May 08, 2017
Abbreviations: ESOD, eerythrocytic superoxide; SOD, eerythrocytic superoxide; CP, ceruloplasmin; ALP, alkaline phosphatase LDH, lactate dehydrogenase; ROS, reactive oxygen species

\section{Introduction}

Malaria continues to be a major public health issue. In 2015 , an estimated 214 million malaria cases occurred, leading to almost 438,000 deaths. ${ }^{1}$ Malaria is one of the most common diseases in Republic of Yemen. Malaria cases in the country are registered all over the year, and a majority of the Yemeni population $(65 \%)$ is exposed to malaria transmission, with $43 \%$ being at high-risk of acquiring the infection ${ }^{2} P$. falciparum, the most dangerous species is the major Plasmodium species in Yemen with only minimal cases caused by P. vivax. ${ }^{3}$

The parasitic infections such as malaria in host organisms often lead to changes in hepatic, erythrocyte activity and oxidative stress condition which are a disturbance in the balance between the productions of reactive oxygen species (ROS) and antioxidant defense. ${ }^{4}$ It is also known that erythrocytes are equipped with antioxidant enzymes that could protect them against damage. ${ }^{5}$ Adapting to the oxidative stress exerted by the host immune response against malaria infection, $P$. falciparum has developed an elaborate reduction-oxidation (redox) system to maintain adequate antioxidant defense throughout its complex life cycle. ${ }^{6}$
Enzymes biocatalysts are involved in all chemical transformation reactions in the body. It may even decrease the amount of free energy needed to activate a specific reaction for the body function. An enzyme may favor the production of only one of the products, where, more than one product is possible in a reaction. Enzyme activities can be affected by molecules produced by the attack of any disease such as malaria, as well as by any dysfunction in metabolic pathways. ${ }^{7}$

The combination of acute hepatocellular injury and red cell haemolysis induced by the invading merozoites may account for the increase in serum ALP and LDH activity during this infection. Therefore a serum ALP and LDH activities is a potentially valuable enzymatic marker of acute malarial infection. ${ }^{8}$ Antioxidants are compounds that are involved in effective scavenging of free radicals and in suppressing the actions of reactive oxygen substances. Antioxidant barriers are extensively distributed and include both enzymatic and non-enzymatic systems. The most important enzymatic antioxidants are superoxide dismutase, glutathione peroxidase and catalase. Nonenzymatic factors that may function as antioxidants are reduced glutathione, vitamin $\mathrm{C}$, vitamin $\mathrm{E}, \beta$-carotene, ceruloplasmin and bilirubin. ${ }^{9}$ These enzymatic and non-enzymatic antioxidant systems are necessary for sustaining life by maintaining a delicate intracellular redox balance and minimizing undesirable cellular damage caused by ROS. ${ }^{10}$ Therefore, we aimed to estimate antioxidants and enzyme activities such as levels in serum of patients with Plasmodium falciparum malaria to compare the ones in the healthy subjects in order to estimate the possible relationship between these parameters. 


\section{Materials and methods}

\section{Selection of subjects}

This study was carried out between October 2014 to March 2015 in the endemic area in Abbs districts. Patients were selected from the Malaria Center in Abbs Rural Hospital, in Hajjah Governorate, Yemen. The study population consisted of 60 patients, who were reported ill with fever (temperature $>37.5^{\circ} \mathrm{C}$ ), headache, vomiting, chills, diarrhea, and other clinical signs and symptoms of malaria as previously documented. ${ }^{11}$ Patients included both sex adults between the ages of 18 and 48 years. Thirty (30) healthy subjects who were symptomatic and negative for $P$. falciparum in their peripheral blood were used as control individuals.

\section{Collection hemolysate and preparation of erythrocyte}

An aliquot of $5 \mathrm{ml}$ of venous blood samples were collected randomly from malaria patients and normal healthy subjects. $1 \mathrm{ml}$ of the blood was slowly ejected into EDTA containing tubes for malaria parasite tests blood, while the rest was left for about $30 \mathrm{~min}$ to coagulate. Samples were centrifuged at $1500 \mathrm{rpm}$ for 15 minutes. Serum was transferred into Bijou bottle and stored frozen until required for the estimation of CP, Vit. C, ALP, LDH. Erythrocytes were washed three times in cold phosphate-buffered saline, followed by re-centrifugation and removal of the supernatant fluid and the buffy coats. The crude red cells were lysed in nine volumes of ice-cold distilled water to prepare a $10 \%$ erythrocyte hemolysate and used for the estimation of superoxide dismutase (SOD).

\section{Estimation of ceruloplasmin (CP)}

$\mathrm{CP}$ was estimated according to the method of Houchin. ${ }^{22}$ : In Cuvette format: add $0.5 \mathrm{ml}$ of $0.1 \% \mathrm{p}$-phenylene-diamine reagent, $100 \mathrm{ml}$ serum was added and mix, leave at $37 \mathrm{oC}$ for 15 minutes, Then $1.25 \mathrm{ml}$ sodium azide was added and OD is measured at $525 \mathrm{~nm}$; Calculate CP activity $=150 \times$ OD525 nm mg/dl.

\section{Estimation of ascorbate (Vitamin C)}

Serum total ascorbate was estimated by the 2 , 4-dinitrophenylhydrazine (DNPH) and method reported by McCormick and Green. ${ }^{13}$

\section{Estimation of superoxide dismutase (SOD)}

SOD activity was determined based on its ability to inhibit the autoxidation of epinephrine in alkaline conditions as described by Misra \& Fridovich ${ }^{14}$ Sigma SOD enzyme was used as standard. OD was measured at $480 \mathrm{~nm}$. The hemoglobin content of the erythrocytes was determined in hemolysate using hemoglobin kit (SPECTRUM) by spectrophotometry.

Estimation alkaline phosphatase (ALP) lactate dehydrogenase (LDH): ALP estimated by Roche/ Hitachi -U.S. Acobasc systems. LDH estimated by kits commercial.

\section{Statistical Analysis}

Data was entered into program of Microsoft Excel and statistic was applied. The data were expressed as mean \pm SE. The results were analyzed statistically using column statistics and one t- tests. Correlation among the investigated parameters was tested by curves and regression using linear regression to test departure from linearity with runs test. These analyses were carried out using computer statistics Prism 3.0 Package (Graph and Software, Inc, San Diego, USA). The minimum level of statistical significance was set at $\mathrm{P}<$ $0.05,0.01$

\section{Ethical considerations}

Ethical approval was given by the Hospital Management and Center of Malaria in Abs area.

\section{Results}

The patients with $P$. falciparum infection showed marked increase of the levels of $\mathrm{CP}(\mathrm{p}<0.001)$ as compared to healthy control subjects (Table 1). The lysate SOD levels were decreased significantly $(p<0.0001)$ as compared to healthy control subjects (Table $1 \&$ Figure 1). Serum vitamin $C$ was decreased significantly $(p<0.0001)$ as compared to healthy subjects (Table $1 \&$ Figure 1 ). In contrast, Serum ALP and LDH levels of patients with Plasmodium falciparum malaria were significantly higher $(\mathrm{p}<0.0001)$ than the healthy control subjects group (Table $2 \&$ Figure 2 ).

Table I Antioxidants of falciparum malarial infected and healthy subjects

\begin{tabular}{lll}
\hline & Patients with malaria & Healthy subjects \\
\hline Parameters & $\mathrm{N}=60$ & $\mathrm{~N}=30$ \\
$\mathrm{CP} \mathrm{mg} / \mathrm{dl}$ & $89.97 \pm 3.42^{* *}$ & $70.69 \pm \mathrm{I} .37$ \\
SOD U/min g Hb & $7.66 \pm 0.32^{* * *}$ & $19.64 \pm 0.50$ \\
Vit. C mg/dl & $0.41 \pm 0.01 * * *$ & $0.83 \pm 0.07$ \\
\hline
\end{tabular}

$* *=p<0.001, * * *=p<0.0001$.

Table 2 Antioxidants of falciparum malarial infected and healthy subjects.y

\begin{tabular}{lll}
\hline & Patients with malaria & Healthy subjects \\
\hline Parameters & $\mathrm{N}=60$ & $\mathrm{~N}=30$ \\
$(\mathrm{ALP}) \mathrm{U} / \mathrm{L}$ & $\mathrm{I} 5 \mathrm{I} .4 \pm 8.85 * *$ & $108.2 \pm 6.80$ \\
$(\mathrm{LDH}) \mathrm{U} / \mathrm{L}$ & $253.1 \pm 3.92 * * *$ & $\mathrm{I} 65.7 \pm 6.5 \mathrm{I}$ \\
\hline
\end{tabular}

$* *=p<0.001, * * *=p<0.0001$

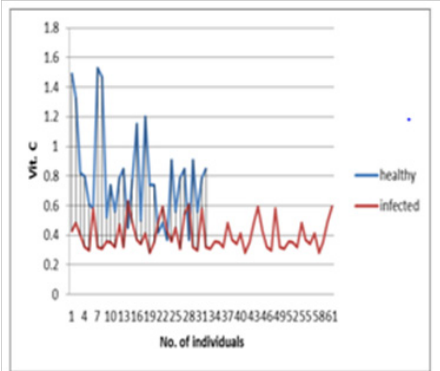

A

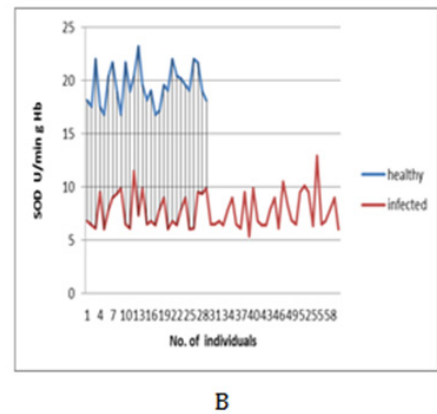

B
Figure I Effect of $P$. falciparum malaria infection on (A) Vit. C and (B) SOD in patients.

\section{Discussion}

We have found in our study highly significant decrease in erythrocyte antioxidants, superoxide dismutase (SOD). The mean erythrocyte concentration of SOD was significantly lower in patients than healthy subjects. Several studies reported reduction of SOD activities of erythrocytes in patients with malaria, ${ }^{15-18}$ which confirms results obtained. This affirms its role as an antioxidant, where levels decreased in an effort to offset the oxidant stress. SOD upgrades endothelial cell damage triggered by adherent parasitized $\mathrm{RBCs}$, underscoring their probable therapeutic benefit as endothelial cell protectors ${ }^{19}$ Pabon et al..$^{20}$, have reported increase in SOD and glutathione peroxidase activity in patients with non-complicated malaria. 

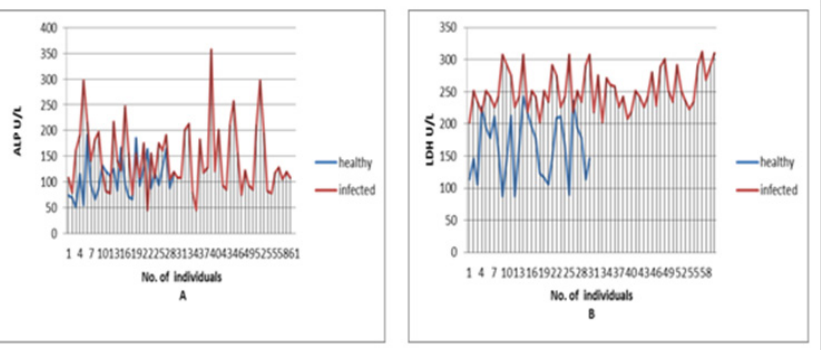

Figure 2 Effect of $P$. falciparum malaria infection on (A) ALP and (B) LDH in patients.

In our findings the levels of $\mathrm{CP}$ in Plasmodium falciparum malaria are significantly increased as compared to the normal healthy controls. Das et al. ${ }^{21}$, have reported high levels of CP in both symptomatic and asymptomatic malaria. Kamble et al. ${ }^{22}$ have reported high levels of $\mathrm{CP}$ in both Plasmodium falciparum malaria and Plasmodium vivax malaria. The significant rise in serum $\mathrm{CP}$ of malaria infected patients may stimulate liver in spite of its pathogenic case, due to malaria infection, to synthesize an excess of CP. The rise of serum $\mathrm{CP}$ during malarial infection may prevent Fenton reaction where the $\mathrm{Fe}+++$ without formation of hydroxyl free redicals. This result suggests that in malarial patients, $\mathrm{CP}$ was activated to contract the production of hydroxyl free radicals. Furthermore, the level of vitamin $\mathrm{C}$ was significantly decreased in malaria subjects when compared with the healthy subject control $(\mathrm{P}<0.0001)$. This finding is similar to previous studies. ${ }^{23}$ The decrease in antioxidant Vitamin $\mathrm{C}$ in the patient groups might be due to counteract increase oxidative stress during acute phase. Supporting to this interpretation the present data showed negative significant correlation between LPO and vitamin C, and this may explain how important vitamin $\mathrm{C}$ is in face of damage resulting from oxidative stress situations.

The liver damage may have been caused by the free radicals generated by the $P$. falciparum parasite. The levels of hydroxyl and peroxide radicals induced by $P$. falciparum parasites may be responsible for the changes in the enzyme levels. ${ }^{24}$ The result of this study shows also significant increase in the serum ALP activity in malaria. ${ }^{25}$ patients. Our results are consistent with other studies which reported that majority of the patients show elevation in serum activities of ALP indicating liver damage as described by several studies. ${ }^{26-28}$ The observed elevation in serum ALP activity is an indication that the hepatic stage of the parasite's life cycle occur in its human host and is accompanied by significant perturbation in the hepatocytes membrane leading to leakage of this enzyme out of the liver cells. The increased serum ALP activity among the patients indicates that the liver stage of Plasmodium falciparum malaria infection is accompanied by a perturbation of the host hepatocytes pathways and damage to hepatocytes membrane leading to leakage of this enzyme out of the liver cells.

Also, this elevation of ALP may be indicator of cholestasis as causative effect of malaria infection. This is consistent with a previous study, ${ }^{29}$ which linked between the serum elevation of ALP activity and cholestasis. Various authors have reported close relationship between incidence of severe malaria and liver damage characterized by jaundice. ${ }^{30,31}$ LDH activity is present abundantly in tissues (liver, $\mathrm{RBC}$ ) which get infected by malaria parasite during completion of asexual cycle. So, raised LDH level may be considered as an evidence for $P$. falciparum infection. Being rich sources of LDH, the acute liver injury and red blood cell destruction will be followed by the release of LDH into the circulation. This finding has important implications because it highlights the potential of using serum LDH activity as an index in the monitoring of acute $P$. falciparum malaria infection, ${ }^{32}$ particularly when all other possible causes of increased serum LDH levels have been observed. ${ }^{8}$ In our study, LDH was increased in $P$. falciparum affected patients as compared to healthy subjects. The increased LDH concentrations among patients with malaria were reported by several authors. ${ }^{8,33,34}$ The observed increase in serum LDH activity in this study may be responsible for acute $P$. falciparum malaria infections during the attack of the liver and RBC cells by sporozoites. The parasite may exert anoxia (Hypoxia) in hepatic tissue which may cause the significant rise in LDH. This interpretation was based on the authors who associated between the rise in LDH and hypoxia. ${ }^{35,36}$

\section{Conflicts of interest}

There is no conflict of interest.

\section{Acknowledgements}

None.

\section{Funding}

None.

\section{References}

1. WHO. World malaria report. Geneva, Switzerland. 2015:280

2. Alkadi HO, Al Maktari MT, Nooman MA. Chloroquineresistant Plasmodium falciparum local strain in Taiz Governorate, Republic of Yemen. Chemotherapy. 2006;52(4):166-170.

3. Abdulsalam MQA, Mohammed AKM, Ahmed AA, et al. Clinical situation of endemic malaria in Yemen. Trop Biomed. 2010;27(3):551558

4. Droge W. Free radicals in the physiological control of cell function. Physiol Rev. 2002;82(1):47-95.

5. Amy M, Gelasius M, Anuraj SH, et al. Antioxidant status and acute malaria in children in Kampala Uganda. Am J Trop Med Hyg. 2001;65(2):115-119

6. Bozdech Z, Ginsburg H. Antioxidant defense in Plasmodium falciparum data mining of the transcriptome. Malar J. 2004;323.

7. http://en.wikipedia.org/wiki/Enzym.

8. GarbaI H, Ubom GA. Total serum lactate dehydrogenase activity in acute Plasmodium falciparum malaria infection. Singapore Med $J$. 2005;46(11):632-634.

9. Murray RK, Mayes AA, Granner DK, et al. Harper's Biochemistry (in Turkish) p. 183. Translated by Menteş G and B. Ersöz. BarIş Printing House, Istanbul, Turkey. 1993.

10. Duuračková Z. Some current insights into oxidative stress. Physiol Res. 2010;59(4):459-469.

11. WHO. Severe falciparum malaria. Trans $R$ Soc Trop Med Hyg. 2000;94:S1-S90.

12. Houchin J. Methods of determination of serum ceruloplasmin level. Am J Biochem. 1958;13:22-41

13. McCormick DB, Green HL. Vitamins. In: Burtis CA and Ashwood ER (Eds.), Tietz Textbook of Clinical Chemistry. $\left(2^{\text {nd }}\right.$ edn), WB Saunders, Philadelphia, USA. 1974:1275-1316.

14. Misra HP, Fridovich I. The role of superoxide anion in the antioxidant of epinephrine and a simple assay for superoxide dismutase. $J$ Biol Chem. 1972;247(10):3170-3175. 
15. Becker K, Tilley L, Vennerstrom JL, et al. Oxidative stress in malaria parasite-infected erythrocytes: host-parasite interactions. Int J Parasitol. 2004;34(2):163-189.

16. Narsaria N, Mohanty C, Das BK, et al. Oxidative stress in children with severe malaria. J Trop Paed. 2012;58(2):147-150.

17. Bilgin R, Yalcin MS, Yucebilgic G, et al. Oxidative stress in vivax malaria. Korean J Parasitol. 2012;50(4):375-377.

18. Tyagi A, Tyagi R, Vekariya R, et al. Study of antioxidant enzymes, MDA and lipid profile in cerebral malaria. Indian Journal of Clinical Practice. 2013;23(12):823-825

19. Ackerman HC, Beandry SD, Fairhurst RM. Antioxidant therapy: reducing malaria severity? Crit Care Med. 2009;37(2):758-762.

20. Pabon A, Carmona J, Burgos LC, et al. Oxidative stress in patients with non-complicated malaria. Clin Biochem. 2003;36(1):71-78.

21. Das BS, Thurnham DJ, Das DB. Influence of malaria on Markers of Status in children: Implication for interpreting iron status in malaria endemic communities. Br J Nutr. 1997;78(5):751-760.

22. Kamble P, Bhagwat V, Trivedi DJ, et al. Comparative study of free radical activity in Plasmodium falciparum and Plasmodium vivax malaria patients. IJPBS. 2011;2(4):B99-B102.

23. Javeed T, Mustafa G, Khan I, et al. Secondary defense antioxidant status of vitamin C, vitamin E and GSH in malaria, caused by Plasmodium falciparum and Plasmodium vivax. Pak J Pharm Sci. 2011;24(2):103107.

24. Nnodium JK, Nwanjo HU, Opara. Blood glucose level and liver enzymes activities in malaria patients in Owerri. $J$ Med Lab Sci. 2010;1:7-9.

25. Maegraith B. Aspects of the pathogenesis of malaria. Southwest Asian J Trop Med Pub Health. 1981;12:251-267.

26. Oyewole IO, Senusia S, Mansaray M. Plasmodium falciparum-induced kidney and liver dysfunction in malaria patients in Freetown, Sierra Leone. Sierra Leone J Biomed Res. 2010;2(1):70-74.
27. Onyesom I, Onyemakonor N. Levels of parasitaemia and changes in some liver enzymes among malarial infected patients in Edo-Delta Region of Nigeria. Cur ResJBiol Sci. 2011;3(2):78-81.

28. Amir F, Ali MS, Chin KY, et al. Serum enzymes activitites in Plasmodium falciparum infection in South Pakistan. Arch Pharma Pract. 2011;2(2):54-56.

29. Alvarez AM, Mukherjee D. Liver Abnormalities in cardiac diseases and heart failure. Int J Angiol. 2011;20(3):135-142.

30. Mishra S K, Mohanty S, Das BS. Hepatic changes in Plasmodium falciparum malaria. Indian J Malaria. 1992;29(3):167-171.

31. Dondorp AM, Day NP. The treatment of severe malaria. Trans $R$ Soc Trop Med Hyg. 2007;101:633-634.

32. Khosya S, Meena R, Meena H. Study of total serum lactate dehydrogenase activity as an indirect evidence of acute Plasmodium falciparum infection. IOSR J Pharm Biolo Sci. 2012;1(2):1-3.

33. Pir MA, Devrajani BR, Baloch S, et al. Serum enzyme activities in patients with vivax malaria and falciparum malaria. International Journal of Multidisciplinary Sciences and Engineering. 2012;3(11):3134.

34. Kumer IH, Suratikiti S, Kumer PH, et al. Serum total LDH activity- A useful tool in aiding differential diagnosis of malaria. J Advance Res Biol Sci. 2013;5(1):25-27.

35. Teng CL , Young JH , Hsu SL, et al. Lactate Dehydrogenase, no Vascular Endothelial Growth Factor or Basic Fibroblast Growth Factor, Positively Correlates to Bone Marrow Vascularity in Acute Myeloid Leukemia. J Chin Med Assoc. 2006;69(11):534-537.

36. Wise DR, Ward PS, Shay JE, et al. Hypoxia promotes isocitrate dehydrogenase-dependent carboxylation of $\alpha$-ketoglutarate to citrate to support cell growth and viability. Proc Natl Acad Sci USA.2011;108(49):19611-19616. 